\title{
Einiges zur Flora von Polen, insbesondere des Städtchens Łosice.
}

Von Ferdinand Karo.

(Sohins.)

Pyrola chlorantha Sw. Wald, Dorf Zakize, sparsam.

- minor L. Wald, Dori Zakrze, Chotycze, sparsam.

- uniflora L. Sehr häufig in Walde bei Zakrze.

- rotundifolia L. Laubwald, Chotycze, hïufig.

Plantago media L. Wiesen bei Losice.

- lanceolata L. Wiesen, Starostwo.

- arenaria W. K. Sandboden an der Chaussée nach Siedlec bei Wyczołki, Głuchów.

Platanthera bifolia Rhb. Laubwald, Chotycze, häufig.

Poa pratensis L. var. angustifolia. Laubwald, Chotyeze.

Polygala rulgaris L. Auf Waldwiesen, Dorf Chotycze.

- comosa Schk. An einem Damme, Starostwo.

Polygonum axiculare L. Brachfelder.

- Convolvulus L. Im Getreide. Nowo Siedlec, Dorf.

- Bistorta L. Sumpfige Wiesen, häufig, Dorf Artych, Patków.

- Persicaria L. Im Getreide, Nowo Siedlec.

Polyganatum officinale All. Laubwald, Chotycze, sparsam.

Potentilla Anserina L. An Wegen, Starostwo.

- alba L. Laubwald, Dorf Chotycze.

- argentea L. An Wegen, Dämmen bei Losice, geinein.

- cinerea Chaix. An Dämmen, Starostwo.

- Tormentilla Schrk. Wälder, Chotycze, Zakrze.

- Güntheri Pohl. Für unsere Flora neu, an einem kleinen Damme, Starostwo. Um Warschau suchte ich sie vergebens.

Primula veris L. Laubwald, Dorf Chotycze, Stok bei Siedlec.

Prunella vulgaris L. Wiesen an Dämmen.

Prunus spinosa L. Wälder, Chotycze, Zalirze, Stok.

- Padus L. Wälder, Majówka.

Pulicaria vulgaris Gärtn. Ueberschwemmte Orte, Polinów bei Losice, Siedlec, Nowa wieś.

Pulmonaria azurea Bess. Laubwald, Dorf Chotycze an der Chaussée nach Siedlec, Osada Mordy, Dorf Stok.

Ranunculus repens L. Sumpfige Wiesen, Starostwo.

- Philonotis Ehrh. Lehmige Getreidefelder, Dorf Lipiny, Biernaty.

- polyanthemos L. Im Walde bei Dorf Chotycze in grosser Menge, wie auch auf Wiesen vor diesem Walde.

- bulbosus L. An einem kleinen Damme, Starostwo, sehr sparsam, sonst nicht bemerkt.

- sceleratus L. Am Rande von Teichen bei Starostwo.

Rhinanthus major Rchb. Wiesen, häufig.

- minor Ehrch. 
Rumex Acetosa L. Wiesen.

- Acetosella L. Brachfelder, Wiesen, läufig.

Salix repens L. Auf Rainen, Dorf Chotyeze.

Salvia pratensis L. Laubwald, Clootycze, an dem Wege nach dem Dorl' Woźnili.

Sambucus nigra L. In Gürten angepflanzt.

Saxifraga granulata L. Aul Rainen und am Rande des Waldes bei Chotycze.

- tridactylites L. Auf einer Wiese, Starostwo.

Senecio palustris D C. Aul torfigem Sumpfboden, Torfstich bei Starostwo, Polinów, Siedlec, hăufig.

- vulgaris L. Schutt an Zidunen.

- Jacobaea L. An Dämmen, Starostwo, vereinzelt.

Scabiosa ochroleuca L. Sonnige Waldhügel Zakrze, Chotycze.

Scirpus sylvaticus L. Am Ufer der Toczna.

Scleranthus perennis L. Auf Getreidefeldern häufig.

Scorzonera humilis L. Auf sandigen Waldstellen Dorf Stok bei Siedlec, aber sehr sparsam.

Scrophularia nodosa L. Gebüsch am Ufer der Toczna, an Teichen, Starostwo, Artych.

Scutellaria galericulata L. An Gräben der Chaussée nach Siedlec, bei dem Dorfe Stok.

Serratula tinctoria L. Wälder, Chotycze sehr sparsam, häufig bei Stok. Silene nutans $\mathrm{L}$. Laubwalder, Chotycze, selten,

- Otites Sm. An eimem Damme bei Patków.

Sinapis arvensis L. Auf Getreidefeldern sehr häufig.

Sisymbrium Thalianum Gay. Auf Brachfeldern bei Chotycze.

- officinale Schl. Auf Schutt, Wegen.

Solanum nigrum L. An Gartenzäunen.

Sonchus arvensis L. v. uliginosus. In Getreidefeldern häufig, Starostwo

Solidago Virgo aurea L. An der Chaussée nach Siedlec.

Spergula Morisonii Bes. Sandige Getreidefelder. Lipiny, Zakrze häufig, Starostwo.

- rubra Presl. Getreidefelder, Zalirze.

Spiraea Ulmaria L. Sumpfige Wiesen im Gebüsch an der Chaussée nach Siedlec bei Dorf Stok.

Stachys recta L. Laubwald, Chotycze selten.

- palustris. An der Toczna, Gebüsch.

Stellaria graminea L. Wiesen, Dorf Artych, häufig.

- media Vill. Auf Aeckern.

- Holostea L. Laubwald, Cholycze, nicht häufig.

- glauca With. Sumpfige Wiesen, Artych.

Succisa pratensis Mnch. Waldwiesen, Szawły, Stock.

Symphytum officinale L. Wiesen, häulig.

Tanacetum vulgare L. An der Chaussée nach Siedlec schr zahlreich, sonst um dosice nirgends bemerist.

Taraxacum officinale W eb. An Dämmen.

Thesium ebracteatum Hayn. In Gebüsch an dem Chausséegraben 
hinter Osada Mordy schr zahlreich, sparsamer im Laubwalde bei Chotycze, scheint für Polen eine sebr verbreitete Pflanze zu sein. Thalictrum minus L. Auf Getreidefelderı, auch auf einer Wiese bei Stok.

Thlaspi arvense L. Getreidefelder, häufig.

Thymus Serpyllum L. Wailder, Sandboden gemein.

Trientalis europaea L. Laubwald bei Chotycze, Lysów.

Trifolium repens L. Aul Wiesen.

- agrarium L. v. aureum Pol. An einem Damme bei Zalsre, zahlreicher im Walde bei Chotycze.

- montanum L. häufig an Wiesen, Wegen.

- alpestre L. Waldwiesen, Dorf Chotycze.

- arvense L. Getreidefelder, sehr gemein.

- fragiferum L. Wiesen an Gräben, feuchten Orten, sehr gemein. Trollius europaeus L. Auf einer Sumpfwiese an der Chaussée nach Siedlec bei Dorf Stock sparsam.

Tussilago Farfara L. An der Toczna.

Vlmus effusa L. Angepflanzt.

Urtica urens L. Schutl, Zäune.

- dioica L. Züunen, Gebüsch.

Vaccinium Myrtillus L. Wälder um Losice.

- Vitis Idaea L. Wälder, Chotycze, Stok.

- uliginosum L. Sumpige Wälder, Dorf Stok.

Valeriana officinalis L. Gebüsch an sumpfigen Stellen bei dem Dorfo Nowo Siedlec sparsam.

Valerianella olitoria Mnch. Auf einer Wiese bei Patlów vereinzelt. Verbascum phoeniceum L. An Feldrainen, Hügeln, in der ganzen Umgegend sehr gemein.

Veronica triphyllos L. Brachfelder, gemein.

- serpyllifolia L. Wiesen, Waldboden, läufig.

- officinalis L. Wälder, Zakrze.

- Beccabunga L. Torfstich bei Starostwo an einem Graben.

- persica Poir. Auf Aeckern, Polinów, häufig.

- polita Fr. Auf Getreidefeldern, Rudniki.

Viburnum Opulus L. Laubwald, Chotycze, gemein.

Vicia sepium L. Gebüsch, Laubwald, Chotycze, Pathów.

- villosa Rth. Getreidefelder, Biernaty, Lipiny.

- angustifolia Rth. Wiesen.

Viola canina L. Waldorte an Wegen, Zakrze, Chotycze.

- canina var. lucorum. Zakrze, sparsam.

- tricolor L. Brachfelder.

- arenaria L. Waldboden bei Dorf Zakrze, sparsam.

Xanthium Strumarium L. An Wegen, Zäunen um und in Losice sehr gemein.

Losice, am 20. Febr. 1871. 